\title{
Management of patients with stress urinary incontinence after failed midurethral sling
}

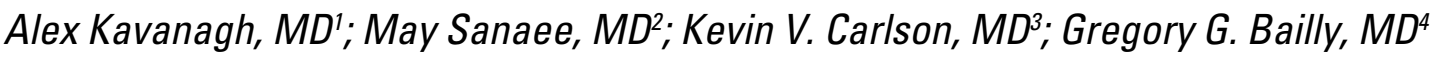

'Department of Urologic Sciences, University of British Columbia, Vancouver, BC; ${ }^{2}$ Department of Obstetrics and Gynecology, University of British Columbia, Vancouver, BC; ${ }^{3}$ Section of Urology, Department of Surgery, University of Calgary, Calgary, AB; ${ }^{2}$ Department of Urology, Dalhousie University, Halifax, NS; Canada

Cite as: Can Urol Assoc J 2017;11 (6Suppl2):S143-6 http://dx.doi.org/10.5489/cuaj.4610

\section{Abstract}

Surgical failure rates after midurethral sling (MUS) procedures are variable and range from approximately $8-57 \%$ at five years of followup. The disparity in long-term failure rates is explained by a lack of long-term followup and lack of a clear definition of what constitutes failure. A recent Cochrane review illustrates that no high-quality data exists to recommend or refute any of the different management strategies for recurrent or persistent stress urinary incontinence (SUI) after failed MUS surgery. Clinical evaluation requires a complete history, physical examination, and establishment of patient goals. Conservative treatment measures include pelvic floor physiotherapy, incontinence pessary dish, commercially available devices (Uresta ${ }^{\circledR}$, Impressa ${ }^{\circledR}$ ), or medical therapy. Minimally invasive therapies include periurethral bulking agents (bladder neck injections) and sling plication. Surgical options include repeat MUS with or without mesh removal, salvage autologous fascial sling or Burch colposuspension, or salvage artificial urinary sphincter insertion. In this paper, we present the available evidence to support each of these approaches and include the management strategy used by our review panel for patients that present with SUI after failed midurethral sling.

\section{Introduction}

With shorter recovery times and comparable efficacy, the midurethral sling (MUS) has surpassed the Burch colposuspension as the "gold standard" treatment for stress urinary incontinence (SUI); however, some patients will not be cured after MUS surgery and currently, there is no consensus on how to manage these patients. This constitutes a major problem not only for the patient, but also for the clinician who is faced with choosing a second surgical procedure with the best possible outcome. Options can include placement of a second synthetic MUS or an autologous sling, such as a pubovaginal sling (PVS). Furthermore, there is no consensus about whether the previously inserted MUS should be excised or if a second tape should simply be placed over the existing tape. The decision on whether to use a sling inserted via an alternative route (retropubic vs. transobturator) also has not been addressed. ${ }^{1}$

Surgical failure rates for MUS are variable and range from approximately $8-57 \%$ at five years of followup. A recent Cochrane review evaluated efficacy of MUS with data contributed by 8652 women. In the short-term (up to one year), the rate of subjective cure of transobturator (TOR) and retropubic (RPR) MUS are similar (relative risk [RR] 0.98; 95\% confidence interval [Cl] 0.96-1.00; 36 trials, 5514 women), ranging from $62-98 \%$ in the TOR group and from $71-97 \%$ in the RPR group. Fewer trials reported medium-term (one to five years) and longer-term (over five years) data, but subjective cure was similar between the groups (RR 0.97; 95\% Cl 0.87-1.09; five trials, 683 women; and RR 0.95; 95\% Cl 0.80-1.12; four trials, 714 women). In the long-term, subjective cure rates were similar and ranged from $43-92 \%$ in the TOR group and from $51-88 \%$ in the RPR group. ${ }^{2}$

The disparity in long-term failure rates is explained by a lack of long-term followup and lack of a clear definition of what constitutes failure. In the above Cochrane review, only four of 55 included studies actually provided information regarding leakage and adverse events at five years. Additionally, "failure" may be defined differently. Failure can include: persistence of bothersome SUI; cure of SUI, but emergence of de novo lower urinary tract symptoms (LUTS); failure to cure SUI and emergence of de novo LUTS or complication (pain, erosion) manifesting postoperatively. These definitions are not mutually exclusive and they can be based on objective testing or subjective history. Furthermore, the term "failure" is used when the symptoms of SUI recur in less than 12 months of the MUS and the term "recurrence" is used if the symptoms return over 12 months later. ${ }^{3}$ 
Kavanagh et al.

\section{Mechanisms of failure}

MUS failure mechanisms can be broadly categorized as 1) preoperative selection of high-risk patient group; 2) failure to correctly treat the original diagnosis; 3 ) onset of new postoperative voiding dysfunction; and 4) failure of surgical technique.

\section{Preoperative selection of high-risk patient group}

A prospective review of nearly 600 patients was performed to evaluate clinical predictors of treatment failure one year after MUS surgery. Prior SUI surgery (odds ratio [OR] 1.99; $95 \% \mathrm{Cl} 1.14,3.47)$; maximum Q-tip excursion less than $30^{\circ}$ (OR 1.89; 95\% Cl 1.16, 3.05); and pad weight per 10 $\mathrm{g}(\mathrm{OR} 1.06 ; 95 \% \mathrm{Cl} 1.02,1.10)$ were predictors of overall failure. Having concomitant surgery (OR $0.44 ; 95 \% \mathrm{Cl} 0.22$, 0.90 ) was predictive of subjective failure only rather than objective failure. Age per 10 years (OR 1.48; 95\% Cl 1.14, 1.90); Urogenital Distress Inventory score per 10 points (OR 1.09; 95\% Cl 1.02-1.17); and pad weight per $10 \mathrm{~g}$ (OR $1.05 ; 95 \% \mathrm{Cl} 1.01,1.10)$ were predictive of objective failure compared to subjective failure only. ${ }^{4}$ Anger et al performed a retrospective review of over 1300 patients followed for one year after MUS with a focus of age as a predictor of adverse event. The authors found that women between the ages of 65 and 74 had less postoperative urge incontinence $(12.6 \%$ vs. $20 \%)$, SUI $(7.2 \%$ vs. $10.5 \%)$, and outlet obstruction $(6.6 \%$ vs. $10.5 \%$ ) compared to women over age $75 .^{5}$

\section{Failure to correctly treat the original diagnosis}

The prevalence of urinary incontinence in Canada is $10 \%$, with a distribution consisting of $50 \%$ SUI, $14 \%$ urgency incontinence, and 33\% mixed incontinence. ${ }^{6}$ MUS used for mixed urinary incontinence or urgency incontinence have lower success rates than when used for pure SUI. A retrospective review of 760 RPR procedures at eight-year followup demonstrates MUS cure rates of $85 \%$ in women with exclusive SUI compared to only $30 \%$ of those with an original diagnosis of mixed urinary incontinence. ${ }^{7}$

\section{Onset of new postoperative voiding dysfunction}

Risk factors for de novo postoperative voiding dysfunction after MUS include preoperative history of voiding dysfunction (OR 2.76), previous retropubic surgery (OR 2.28), and concurrent reconstructive procedures with MUS (OR 4.88). ${ }^{8}$ For patients with preoperative detrusor overactivity, one-third have resolution of symptoms with placement of a midurethral sling; however, the majority have some persistence of symptoms. Risk factors for persistence include advanced age, increased nocturia, lower bladder capacity $(<450 \mathrm{~mL})$, sling type (favouring TOR route), higher detrusor overactivity pressure, and lower volume at detrusor contraction. ${ }^{9}$

\section{Failure of surgical technique}

Acute failure attributed to intraoperative and perioperative complication after MUS is uncommon. Reoperation rates related to tape insertion or postoperative voiding dysfunction range from $0.8-2.4 \%$. Postoperative acute urinary retention is $0.5-1.6 \%$. Placement of a loosely tensioned sling with insufficient support is difficult to quantify, but likely contributes to the overall rate of acute (up to one year) recurrent SUI of $2-38 \%$ in the TOR group and from $3-29 \%$ in the RPR group. The rate of erosion (graft material in the lumen of the urinary tract or pelvic viscera) and extrusion (exposed graft material in the vagina) is $0.4-1.5 \%$, but data is primarily limited to short-term (less than five-year) followup. ${ }^{2}$

\section{Evaluation}

Clinical evaluation requires a complete history, physical examination, and establishment of patient goals. It is important to establish whether the pattern of stress incontinence is the same as pre-procedure, or different. A voiding diary and structured questionnaire, such as the International Consultation on Incontinence Questionnaire (ICIQ), can enhance the history. The severity of leakage, based on subjective pad usage (and type of pad), in addition to the degree of bother, should be considered. Physical examination is very important and should be performed in the dorsal lithotomy (and/or standing positions), ensuring that the bladder is not empty first. Visualization of the urethra can help demonstrate leakage with Valsalva and distinguish hypermobility from possible intrinsic-sphincter deficiency. A post-void residual can also help identify possible voiding dysfunction. Half-speculum examination in a patient with mesh in place is critical to rule out vaginal extrusion. Cystourethroscopy is indicated for all patients with prior anti-incontinence procedure to evaluate urethral erosion. Finally, urodynamic studies may be indicated to rule out bladder outlet obstruction and/or confirm SUI when the history and examination have not adequately characterized the situation. Transvaginal ultrasound evaluation has been advocated to determine the location of the mesh with respect to the urethral length and lumen, but its clinical use remains questionable.

\section{Management}

Treatment options for SUI after failed MUS include observation, conservative therapy, minimally invasive therapy, and surgical intervention. A recent Cochrane review illustrates that no high-quality data exists to recommend or refute any of the different management strategies for recurrent or persis- 
tent SUI after failed MUS surgery. ${ }^{1}$ As such, we rely primarily on level 4 expert opinion to guide treatment decisions.

\section{Conservative therapy}

Conservative measures include pelvic floor physiotherapy, incontinence pessary dish, ${ }^{10}$ commercially available devices $\left(\right.$ Uresta $^{\circledR}$, Impressa $\left.{ }^{\circledR}\right)$ or medical therapy. Unfortunately, these options have not been well-studied in the context of MUS failure. It is the expert opinion of the reviews that patients with residual or recurrent minimal leakage should be encouraged to pursue conservative measures prior to considering repeat surgery.

\section{Minimally invasive therapy}

Minimally invasive therapies include periurethral bulking agents (bladder neck injections) and sling plication. A retrospective review of 74 patients treated with Durasphere ${ }^{\circledR}$ bladder neck injection after failed MUS was performed by Kim et al. ${ }^{11}$ A success rate of nearly $40 \%$ was reported at five years of followup; however, when compared against a repeat MUS, bladder neck injection has a higher risk of failure at 12 months of nearly $40 \%$ vs. $11 \%$ (OR 3.29; Cl 1.34-9.09). ${ }^{12}$ Overall, with the lack of consistent data and low rates of success, bulking agents should rarely be considered effective in providing durable and effective management of recurrent SUI in most women, with the exception being poor surgical candidates, elderly patients, and those with factors obviating repeat vaginal dissection.

Sling plication refers to tightening an existing MUS mesh. Only five small retrospective series have been published using sling plication after failed MUS, with a pooled success rate of $61 \% .{ }^{13}$ When compared to repeat MUS, plication has a higher risk of failure at 12 months (53\% for sling plication vs. $38 \%$ for repeat MUS). ${ }^{14}$ Of note, higher plication success is found with retropubic slings (88\%) than with TOR slings (45\%). ${ }^{13}$ Due to the lack of data, sling plication cannot be recommended for treatment of recurrent SUI following sling placement and none of the authors employ this strategy.

\section{Surgical intervention}

Surgical options include repeat MUS with or without mesh removal, salvage autologous fascial sling or retropubic (Burch) colposuspension, or salvage artificial urinary sphincter insertion. Repeat MUS has been evaluated by approximately 10 low-quality, small-enrollment, retrospective trials with variable definitions of success, variable types of MUS used in primary and repeat cases, and limited followup. Although it is difficult to draw conclusions from heterogeneous data, the available evidence suggests that: 1 ) repeat MUS surgery is less effective than primary surgery; 2 ) overall success rates vary widely (40-100\%); and 3) there is a suggestion that repeat RPR slings may have superior cure rate compared to repeat TOR slings. ${ }^{1}$ It is the expert opinion of the authors that a retropubic, and not transobturator, MUS should be offered as secondary surgery for a patient who has failed a previous MUS. This is always the case in those who were previously implanted with a TOR, and more selectively in those with a prior RPR (Fig. 1). We feel that urethral hypermobility is key to the efficacy of the MUS. If hypermobility is not present after failed MUS (i.e., urethra

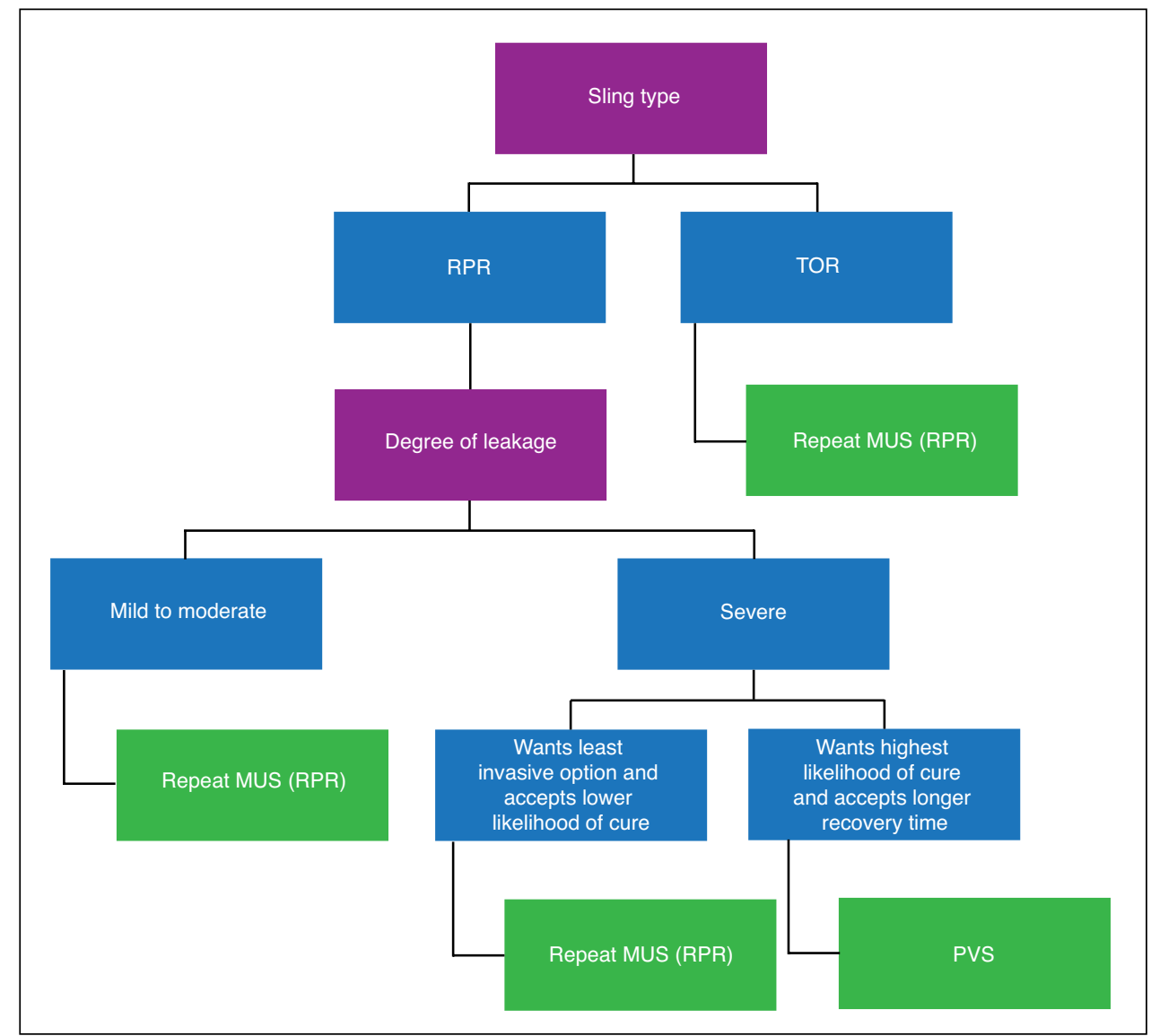

Fig. 1. Suggested management approach for isolated residual or recurrent SUI following a single prior midurethral sling (MUS). PVS: pubovaginal sling; RPR: retropubic; TOR: transobturator. 
Kavanagh et al.

is fixed in position), we support releasing the original MUS prior to placement of secondary RPR.

Although many urologists prefer salvage autologous fascial pubovaginal sling (PVS) after MUS failure, there is a lack of evidence supporting this approach. Recently, Petrou et al published a retrospective review of their experience with 21 patients that underwent salvage autologous fascial sling after failed RPR MUS. At 74 months of followup, 52\% of patients remained dry..$^{15}$ It is our opinion that patients with a mild to moderate degree of leakage benefit from repeat RPR MUS, and those with more severe leakage are more likely to benefit from PVS (Fig. 1). Additionally, our panel suggests that patients with more than one prior failed MUS and isolated recurrent SUI should be offered a PVS (rectus fascia preferred when possible).

There are two small retrospective studies of 13 and 16 patients that underwent open or laparoscopic Burch colposuspension after failed MUS. Objective cure rates were $77 \%$ and $54 \%$, respectively, at a median followup of one year or 24.5 months. ${ }^{16,17}$ Although no studies specifically evaluate artificial urinary sphincter (AUS) insertion after failed MUS, Valeux et al reviewed their experience with 245 female AUS cases. Failed prior continence procedures had been performed in $88.8 \%$. With a mean followup of 6 years, $74 \%$ of the patients used ( 0 or 1 pad/day) and $65 \%$ used no pads. ${ }^{18}$ The authors and expert review panel do not routinely use these approaches; however, they may be appropriate in very select cases.

\section{Conclusion}

$\mathrm{SUI}$ is a common problem, and to date, no treatment leads to a $100 \%$ cure for all patients. As such, a subset of patients will fail surgery and develop recurrence of symptoms. This is frustrating to both patient and provider. It is critical in all such cases to "start over" with a careful history, physical examination, and appropriate ancillary testing to rule out other causes of "failure" aside from recurrent or residual stress leakage. A recent Cochrane review illustrates that no high-quality data exists to recommend or refute any of the different management strategies for recurrent or persistent SUI after failed MUS surgery. In this article, we have summarized the available evidence and attempted to provide expert opinion to guide the reader in managing these challenging cases.

Competing interests: The authors report no competing personal or financial interests relevant to this article.

This paper has been peer reviewed.

\section{References}

1. Bakali E, Buckley BS, Hilton P, et al. Treatment of recurrent stress urinary incontinence after failed minimally invasive synthetic suburethral tape surgery in women. Cochrane Database Syst Rev 2013:CD009407. https://doi.org/10.1002/14651858.cd009407.pub2

2. Ford AA, Rogerson L, Cody JD, et al. Midurethral sling operations for stress urinary incontinence in women. Cochrane Database Syst Rev 2015:CD006375. https://doi.org/10.1002/14651858.cd006375.pub3

3. Smith AR, Artibani W, Drake MJ. Managing unsatisfactory outcome after midurethral tape insertion. Neurourol Urodyn 2011;30:771-4. https://doi.org/10.1002/nau.21090

4. Richter HE, Litman HJ, Lukacz ES, et al. Demographic and clinical predictors of treatment failure one year after midurethral sling surgery. Obstet Gynecol 2011;117:913-21. https://doi.org/10.1097/ AOG.0b013e31820f3892

5. Anger JT, Litwin $M S$, Wang $Q$, et al. The effect of age on outcomes of sling surgery for urinary incontinence. J Am Geriatr Soc 2007;55:1927-31. https://doi.org/10.1111/j.1532-5415.2007.01470.x

6. Beftez $M$, Tu le M, Carlson K, et al. 2012 update: Guidelines for adult urinary incontinence collaborative consensus document for the Canadian Urological Association. Can Urol Assoc J 2012;6:354-63. https://doi.org/10.5489/cuaj.12248

7. Holmgren C, Nilsson S, Lanner L, et al. Long-term results with tension-free vaginal tape on mixed and stress urinary incontinence. Obstet Gynecol 2005;106:38-43. https://doi.org/10.1097/01. AOG.0000167393.95817.dc

8. Molden S, Patterson D, Tarr M, et al. Risk factors leading to midurethral sling revision: A multicentre case-control study. Int Urogynecol J 2010;21:1253-9. https://doi.org/10.1007/s00192-010-1186-1

9. Gamble TL, Botros SM, Beaumont JL, et al. Predictors of persistent detrusor overactivity after transvaginal sling procedures. Am J Obstet Gynecol 2008;199:696el-7.

10. Noblett KL, McKinney A, Lane FL. Effects of the incontinence dish pessary on urethral support and urodynamic parameters. Am J Obstet Gynecol 2008; 198:592e 1-5.

11. Kim J, Wai L, Lucioni A, et al. Long-term efficacy and durability of durasphere urethral bulking after failed urethral sling for stress urinary incontinence. J Urol 2012;187:e552. https://doi.org/10.1016/i. juro.2012.02.1743

12. Gaddi A, Guaderrama N, Bassiouni N, et al. Repeat midurethral sling compared with urethral bulking for recurrent stress urinary incontinence. Obstet Gynecol 2014;123:1207-12. https://doi.org/10.1097/ AOG.0000000000000282

13. Patterson D, Rajan S, Kohli N. Sling plication for recurrent stress urinary incontinence. Female Pelvic Med Reconstr Surg 2010;16:307-9. https://doi.org/10.1097/SPV.0b013e3181ed3fc3

14. Han JY, Moon KH, Park CM, et al. Management of recurrent stress urinary incontinence after failed midurethral sling: Tape tightening or repeat sling? Int Urogynecol J 2012;23:1279-84. https://doi.org/10.1007/s00192-012-1737-8

15. Petrou SP, Davidiuk AJ, Rawal B, et al. Salvage autologous fascial sling after failed synthetic midurethral sling: Greater than three-year outcomes. Int J Urol 2016;23:178-81. https://doi.org/10.1111/ ïu. 13003

16. Abdel-Fattah M, Ramsay I, Pringle $S$, et al. Randomized, prospective, single-blinded study comparing 'inside-out' vs. 'outside-in' transobturator tapes in the management of urodynamic stress incontinence: One-year outcomes from the E-TOT study. BJOG 2010;117:870-8. https://doi.org/10.1111/i.14710528.2010.02544.x

17. Maher $C$, Dwyer $P$, Carey $M$, et al. The Burch colposuspension for recurrent urinary stress incontinence following retropubic continence surgery. Br J Obstet Gynaecol 1999;106:719-24. https://doi.org/10.1111/i.1471-0528.1999.tb08373.x

18. Vayleux B, Rigaud J, Luyckx $F$, et al. Female urinary incontinence and artificial urinary sphincter: Study of efficacy and risk factors for failure and complications. Eur Urol 2011;59:1048-53. https://doi.org/10.1016/i.eururo.2011.03.006

Correspondence: Dr. Alex Kavanagh, Department of Urologic Sciences, Faculty of Medicine, University of British Columbia, Vancouver, BC, Canada; alex.kavanagh@ubc.ca 